\title{
Deconstructing the genetic basis of spent sulphite liquor tolerance using deep sequencing of genome-shuffled yeast
}

\author{
Dominic Pinel ${ }^{1,3}$, David Colatriano ${ }^{1}$, Heng Jiang ${ }^{1,4}$, Hung Lee $^{2}$ and Vincent JJ Martin ${ }^{\text {* }^{*}}$
}

\begin{abstract}
Background: Identifying the genetic basis of complex microbial phenotypes is currently a major barrier to our understanding of multigenic traits and our ability to rationally design biocatalysts with highly specific attributes for the biotechnology industry. Here, we demonstrate that strain evolution by meiotic recombination-based genome shuffling coupled with deep sequencing can be used to deconstruct complex phenotypes and explore the nature of multigenic traits, while providing concrete targets for strain development.

Results: We determined genomic variations found within Saccharomyces cerevisiae previously evolved in our laboratory by genome shuffling for tolerance to spent sulphite liquor. The representation of these variations was backtracked through parental mutant pools and cross-referenced with RNA-seq gene expression analysis to elucidate the importance of single mutations and key biological processes that play a role in our trait of interest. Our findings pinpoint novel genes and biological determinants of lignocellulosic hydrolysate inhibitor tolerance in yeast. These include the following: protein homeostasis constituents, including Ubp7p and Art5p, related to ubiquitin-mediated proteolysis; stress response transcriptional repressor, Nrg1p; and NADPH-dependent glutamate dehydrogenase, Gdh1p. Reverse engineering a prominent mutation in ubiquitin-specific protease gene UBP7 in a laboratory S. cerevisiae strain effectively increased spent sulphite liquor tolerance.

Conclusions: This study advances understanding of yeast tolerance mechanisms to inhibitory substrates and biocatalyst design for a biomass-to-biofuel/biochemical industry, while providing insights into the process of mutation accumulation that occurs during genome shuffling.
\end{abstract}

Keywords: Evolutionary engineering, Genome shuffling, Reverse engineering, Complex trait, Tolerance, Yeast

\section{Background}

Mapping genotype to phenotype for complex traits and using these data for the rational design of biocatalysts is a natural progression in an increasingly sophisticated biotechnology industry. Unfortunately, current technologies do not allow for the rapid creation of industrially relevant microorganisms or the ability to access and understand multigenic phenotypic traits. Traditionally, strain improvement has been based on a repetitive cycle of random mutagenesis and selection to improve the phenotypic traits of industrial microbes [1]. Advanced

\footnotetext{
* Correspondence: vincent.martin@concordia.ca

'Department of Biology, Centre for Structural and Functional Genomics, Concordia University, 7141 Sherbrooke Street West, Montréal, Québec H4B 1R6, Canada

Full list of author information is available at the end of the article
}

DNA sequencing technology now allows for rapid sequencing of the genomes of these industrial strains to identify the mutations that confer improved phenotypes. However, in resequencing the genomes of randomly evolved strains, a small number of potentially productive mutations are often accompanied by a background of non-productive mutations [2-4]. Extensive functional characterizations of individual genotypic variations are therefore needed to unravel which mutations are associated with the phenotype of interest. Furthermore, our ability to deconstruct complex, multigenic traits is still limited. Possible solutions to these problems include sequencing pools of independent mutants [5], backcrossing non-productive mutations prior to genome resequencing, or combining intercrossing with pool sequencing to assign quantitative trait loci [6] in order to hone 
in on productive mutations. Nonetheless, resolving such data into manageable and testable hypotheses can be insurmountably challenging.

In this study, we aimed to mitigate these shortcomings by sequencing a strain created by genome shuffling from a known background strain and tracking mutations throughout the evolving population. This allows for important mutations to be ranked and novel gene targets to be acquired from background mutations. Moreover, genome shuffling (GS) is an alternative to classical strain improvement that is a means to accelerate the evolution of industrial strains in the laboratory and minimize the accumulation of non-productive mutations. The rationale behind GS is to rapidly combine beneficial mutations and cross out deleterious ones, which can be achieved in Saccharomyces cerevisiae by recursive poolwise mating of mutant populations (Figure 1A) [7-10]. This strain engineering technique is particularly powerful to address multigenic, complex phenotypes such as resistance to ethanol, lactic acid, heat and low $\mathrm{pH}$ or production of compounds like tylosin or taxol (reviewed in [11]). Theoretically, the background of non-productive or deleterious mutations can be minimized by attenuating mutagen dosage, screening for parental strains that contain productive mutations, followed by trait-enhanced mutant strain recombination to combine mainly productive mutations into a single strain. Furthermore, by its very nature, GS brings interacting mutations together into single strains. Although the utility of GS has been demonstrated repeatedly through phenotypic observation, the nature of the mutations accumulated during the strain evolution has not been tracked through genome resequencing. Sequencing GS isolates, therefore, should yield access to determinants of multigenic traits at single nucleotide resolution, while minimizing non-productive variation discovery. Tracking mutations throughout the population of genome-shuffled strains can then be used to further increase the possibility of finding productive mutations.

Microbial tolerance to lignocellulosic hydrolysates is a complex, multigenic trait that is of significant importance to a biomass-to-fuel/chemical industry. The pretreatment of lignocellulose to fermentable sugars yields many byproducts that are inhibitory to fermenting yeasts. The main sources of inhibition come from osmotic pressure, reactive oxygen species (ROS) damage or compounds that include furan aldehydes, primarily furfural and 5(hydroxymethyl)-2-furaldehyde (HMF), phenolics and organic acids, especially acetic, formic and levulinic acids [12-16]. The biological factors implicated in the tolerance of yeast to lignocellulose fermentation inhibitors have been reviewed $[12,13,17]$. Ultimately, engineering productive industrial biocatalysts with tolerance traits will be a pervasive biotechnological problem, and rationally engineering these traits will require an understanding of interacting genes and biological processes that affect tolerance. Currently, a lack of knowledge on the multiple cellular processes and genes involved in microbial tolerance to lignocellulosic hydrolysates makes rational engineering of strains resistant to these substrates implausible $[8,18,19]$.

In a previous study [8], we evolved a strain of S. cerevisiae, R57, through genome shuffling (Figure 1A,B) that is capable of survival, growth and ethanol productivity in hardwood spent sulphite liquor (HWSSL), a highly inhibitory lignocellulosic substrate generated by the acid bisulphite pulping process [20,21], to levels of tolerance previously unreported. HWSSL contains sugars, lignosulphonates, inhibitory compounds, residual pulping chemicals, ammonia and sulphite [22] and can contain heavy metal ions (iron, chromium, nickel and copper) that originate from the corrosion of pulping and bleaching equipment $[23,24]$. Some of the major constituents are approximated at the following levels (\% w/v): $0.83 \%$ to $1.45 \%$ hexose sugars, $1.7 \%$ to $2.1 \%$ pentose sugars, $0.18 \%$ to $0.5 \%$ furfural, $0.9 \%$ to $1.0 \%$ acetic acid, $0.5 \%$ to $0.7 \%$ sulphate, $1 \%$ ammonia and $17 \%$ lignosulphonate $[8,25]$. In evolving R57 through GS, it was hypothesized that beneficial, tolerance-conferring mutations were combined through recursive population-wise meiotic recombination, which yielded a progression towards higher tolerance of the hydrolysate displayed with each subsequent round of GS (Figure 1) [8]. Strain R57 showed improved crosstolerance to several known inhibitors of lignocellulosic hydrolysates [8], supporting the theory that R57 harbours multiple tolerance-conferring mutations. In this study, we resequenced the genome of R57 in order to discover the mutations that were accumulated to confer hydrolysate tolerance, combined with profiling the relative abundance of R57 mutations in the heterogeneous parental GS populations to probe for the relative phenotypic effect of each discovered mutation and explore mutation recombination in GS-evolved pools.

This study describes a relatively cost-effective way to explore combinatorial space of productive mutations within a single genome. To our knowledge, this is the first study to resequence the genome of a strain evolved through GS and the first resequencing project for a yeast strain specifically evolved for its tolerance to a complex mixture of inhibitors in lignocellulosic substrates.

\section{Results and discussion}

\section{Genome sequencing of GS-evolved strain R57}

The genome of $S$. cerevisiae strain R57 was resequenced in an effort to pinpoint genetic changes associated with its tolerance to HWSSL. Both the parental haploid CEN. PK113-7D and mutant diploid R57 were sequenced and compared at approximately 100-fold and approximately 350-fold coverage per nucleotide (Additional file 1: Data S1), 


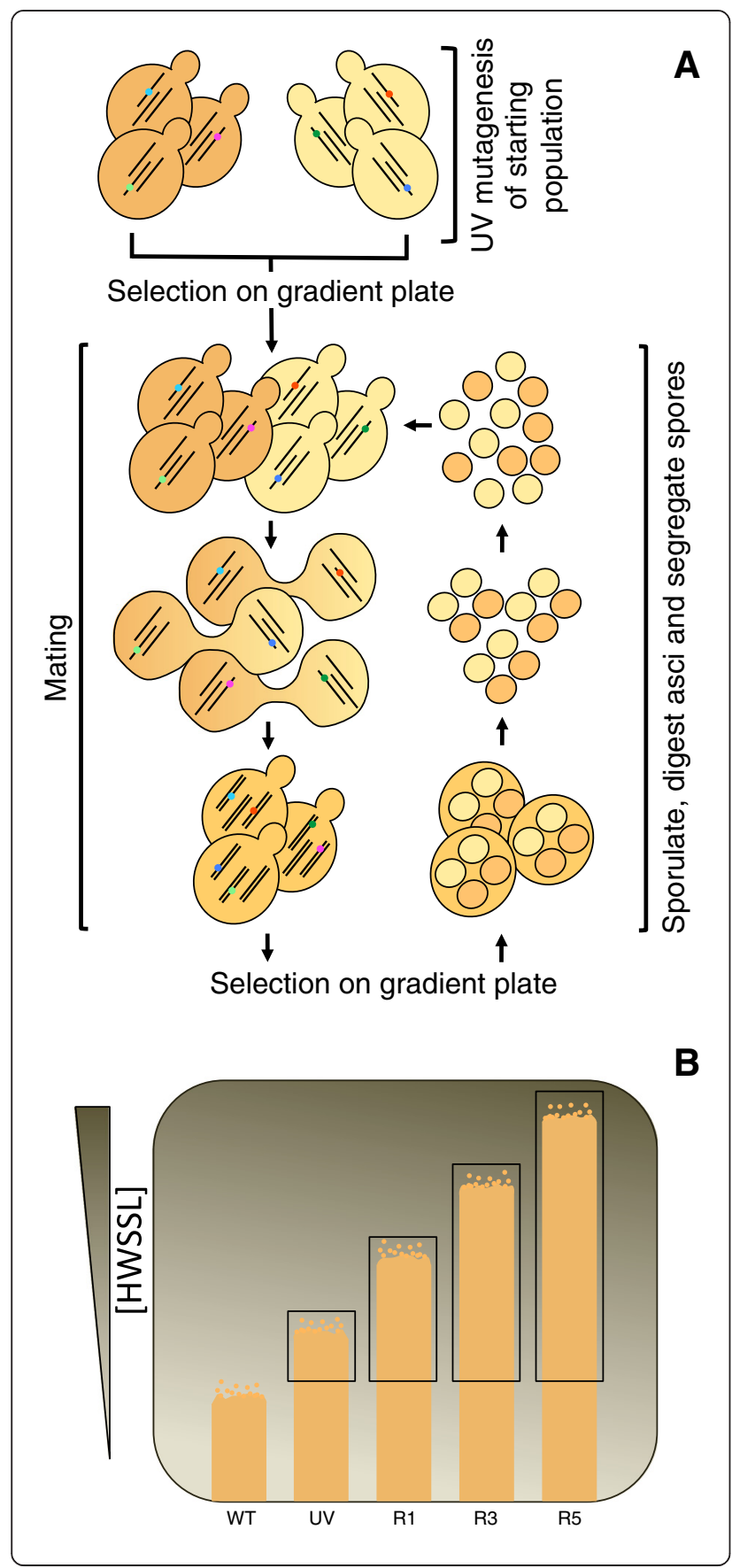

Figure 1 Meiotic recombination-mediated genome shuffling by recursive breeding for HWSSL tolerance. (A) A recursive mating methodology was used to create the HWSSL strains and populations used in this study. Large pools of UV mutants and genome-shuffled populations were screened on HWSSL gradient agar plates prior to each round of shuffling. (B) Portions of each population that showed more tolerance than the reference (WT) (black boxes) were scraped from gradient plates and used for genome shuffling (different rounds of genome shuffling are depicted - round 1 (R1), round 3 (R3) and round 5 (R5)). Initial UV mutant populations (UV) of each

haploid mating type showing enhanced HWSSL tolerance were scraped and used to begin the recursive breeding methodology. Selection on HWSSL gradient plates was carried out between each round of GS in order to enrich the mating pool for strains showing the tolerance phenotype. A portion of each mutant pool (UV through five rounds of GS) was frozen for population sequencing (see 'Results and discussion'). Individual colonies showing the highest tolerance to HWSSL were isolated from the frontier of growth. HWSSL, hardwood spent sulphite liquor.

respectively, which allows for meaningful mutation prediction [2]. The relative level of sequence read coverage per chromosome between the strains is similar and suggests the absence of aneuploidy (Additional file 1: Data S1). Insertion/deletion (indel) and copy number variation (CNV) analysis returned no detectable differences between the wild type (WT) and R57 after visual inspection. All of the mutations discovered from the mutation analysis are single nucleotide polymorphisms (SNPs) and were confirmed by Sanger sequencing.

Twenty-one point mutations were found that could affect at least 17 genes, based on location within open reading frames (ORFs) or untranslated regions (UTRs). These include 16 SNPs affecting 12 ORFs, 14 of which lead to missense mutations, with the remaining 2 leading to silent mutations (Table 1). The five mutations not found within ORFs are all located in $5^{\prime}$ or $3^{\prime}$ UTRs. A heterogeneous SNP lies $43 \mathrm{bps} 3^{\prime}$ of $B C S 1$ and is predicted to be part of the 5' UTR of YDR374C [26] and in the 3' UTR of WIP1 [27]. This mutation is not included in subsequent analyses due to the ambiguity of the affected gene. Gene ontology categories and interaction maps for the affected genes were generated (Additional file 2: Methods and Additional file 3: Figures S1 and S2), and the mutation analysis results are summarized in Table 1.

\section{Predicting protein function and phenotypic effect for missense mutations in R57}

Functional prediction of altered primary protein structure was carried out using the SIFT (Sorting Intolerant From Tolerant) algorithm [28-30] (Table 1). Ten of the 14 missense mutations are expected to affect protein function, with 4 predicted as tolerated by the protein. All but two of the mutations are heterozygous and therefore are expected to have a dominant effect if they contribute to the R57 phenotype. The two homozygous mutations are 
Table 1 Point mutations discovered in GS-evolved strain R57

\begin{tabular}{|c|c|c|c|c|c|}
\hline Gene & Chr & Mutation & Gene function & Genotype & $\begin{array}{l}\text { SIFT } \\
\text { score }\end{array}$ \\
\hline NRG1 & IV & $137 \mathrm{C}>\mathrm{A}(\mathrm{P} 46 \mathrm{Q})$ & Transcriptional repressor, stress tolerance & Homo & 0 \\
\hline$U B P 7^{a}$ & IX & $2466 \mathrm{~T}>\mathrm{A}(\mathrm{N} 822 \mathrm{~K})$ & Ubiquitin-specific protease & Homo & 0.33 \\
\hline$A R T 5^{\mathrm{a}}$ & VII & $454 C>A(L 152 I)$ & $\begin{array}{l}\text { Regulates endocytosis and turnover of cell-surface proteins by targeted } \\
\text { ubiquitination }\end{array}$ & Hetero & 0.17 \\
\hline$S S A 1^{a}$ & I & $91 \mathrm{C}>\mathrm{A}(\mathrm{Q} 31 \mathrm{~K})$ & ATP-ase, protein folding, heat shock, HSP70 & Hetero & 0 \\
\hline$G D H 1^{\mathrm{b}}$ & $\mathrm{XV}$ & $47 \mathrm{C}>\mathrm{T}(\mathrm{S} 16 \mathrm{~F})$ & Glutamate synthesis from ammonia & Hetero & 0 \\
\hline$G D H 1^{\mathrm{b}}$ & & $68 \mathrm{~T}>\mathrm{G}(\mathrm{F} 23 \mathrm{C})$ & & Hetero & 0 \\
\hline$A R O 1^{\mathrm{b}}$ & IV & $1283 \mathrm{C}>\mathrm{T}(\mathrm{S} 428 \mathrm{~F})$ & Catalyzes biosynthesis of chorismate leading to aromatic amino acids & Hetero & 0 \\
\hline$A R O 1^{\mathrm{b}}$ & & $1284 \mathrm{C}>\mathrm{T}$ (Silent) & & Hetero & - \\
\hline STE5 ${ }^{\mathbf{b}}$ & IV & $512 \mathrm{C}>\mathrm{T}(\mathrm{S} 171 \mathrm{~F})$ & Pheromone-response scaffold protein, forms MAPK cascade complex & Hetero & 0 \\
\hline STE5 ${ }^{\mathbf{b}}$ & & $2649 \mathrm{~T}>\mathrm{C}$ (Silent) & & Hetero & - \\
\hline$M A L 11^{\mathrm{b}}$ & VII & $310 \mathrm{C}>\mathrm{T}(\mathrm{P} 104 \mathrm{~S})$ & Alpha-glucoside symporter, with high affinity for trehalose & Hetero & 0 \\
\hline$M A L 11^{\mathrm{b}}$ & & $482 \mathrm{~T}>\mathrm{A}(\mathrm{M} 161 \mathrm{~K})$ & & Hetero & 0.02 \\
\hline$G S H 1^{c}$ & $\mathrm{x}$ & $\mathrm{T}>\mathrm{A}\left(73 \mathrm{bp} 5^{\prime}\right.$ UTR $)$ & Glutamylcysteine synthetase, glutathione biosynthesis & Hetero & - \\
\hline$P B P T^{C}$ & $\mathrm{VII}$ & $\mathrm{T}>\mathrm{C}\left(191 \mathrm{bp} 5^{\prime}\right.$ UTR $)$ & Controls mRNA poly(A), stress granule formation and translation control & Hetero & - \\
\hline FIT3 & $X V$ & $\mathrm{C}>\mathrm{T}\left(42 \mathrm{bp} 3^{\prime}\right.$ UTR $)$ & Iron transport & Hetero & - \\
\hline NOP5 $8^{\mathrm{C}}$ & $X V$ & A $>$ T (25 bp $3^{\prime}$ UTR $)$ & Pre-rRNA processing and rRNA synthesis & Hetero & - \\
\hline YNL058C $^{d}$ & XIV & $7 A>G(K 3 E)$ & Unknown function & Hetero & 0.42 \\
\hline$D O P 1^{d}$ & IV & $40 \mathrm{~A}>\mathrm{T}(\mathrm{N} 14 \mathrm{Y})$ & Endosome to Golgi transport, ER organization, cell polarity and morphogenesis & Hetero & 0.05 \\
\hline TOF2 $^{\mathrm{d}}$ & $X I$ & $2141 \mathrm{C}>\mathrm{T}(\mathrm{S} 714 \mathrm{~L})$ & rDNA silencing, stimulates Cdc14p for mitotic rDNA separation & Hetero & 0.27 \\
\hline$S G O 1^{d}$ & $X V$ & $575 C>A(S 192 Y)$ & Chromosomal segregation and stability & Hetero & 0.03 \\
\hline
\end{tabular}

Bold font represents alleles that gain in frequency over GS evolution. ${ }^{\mathrm{a} G e n e}$ group containing genes that are related to protein homeostasis. ${ }^{\mathrm{b}} \mathrm{Gene}$ group containing genes bearing more than one mutation in R57. 'Gene group containing UTR mutations. ${ }^{\mathrm{d}}$ Gene group containing alleles with limited evidence for a phenotypic linkage to HWSSL tolerance.

located within NRG1 and UBP7. Homozygous mutations at these loci suggest that they have been enriched at a high enough population density during GS evolution that they were able to mate with the opposite mating type and may therefore be important to the HWSSL tolerance trait or that spores were insufficiently segregated during GS (Figure 1A), promoting homozygosity.

Several genes contain multiple mutations in R57. Genes bearing more than one mutation in R57 suggest that the affected gene is important to the phenotype and the mutations have accumulated due to GS evolution and screening. Two missense mutations affect both GDH1 and MAL11. Gdh1p bears two mutations in R57, S16F and $\mathrm{F} 23 \mathrm{C}$. Visual inspection of the mapping alignment shows that the two mutations are never located on the same sequence read, and therefore, both versions of GDH1 are mutated in R57. The close proximity of these two mutations suggests that a mutation in this region of GDH1 may yield a phenotypic trait that has been selected for through GS evolution. The R57 MAL11 gene contains two missense mutations (leading to P104S and M161K). Cloning and sequencing of MAL11 from R57 shows that the two mutations are not located on the same allele. STE5 and ARO1 each bear a second silent mutation that may be present due to close genetic linkage to the productive mutation or lead to a non-obvious phenotypic modification, such as altered mRNA stability, and thereby arise in R57 through selection.

\section{Analysis of mutation loci in GS-evolved heterogeneous populations}

We deeply sequenced the R57 mutation loci within the pooled mutant populations generated during the genome shuffling experiments [8]. The mutation loci were PCR amplified from DNA extracted from the heterogeneous populations and sequenced. There were a total of $3.76 \times 10^{6}$ reads with an average read length of $104 \mathrm{bp}$ and an average fold coverage of $1.35 \times 10^{5}$ reads per nucleotide sequenced. Eighteen SNPs were called (Figure 2). We assessed the frequency of each sequence read that contains a mutation within the total number of sequence reads spanning each locus (Figure 2). These data were used to predict mutations that may have arisen in single parental strains, due to similar prevalence within a population, or may be epistatic, and to probe for changes in SNP frequency between populations that could indicate the relative influence of those alleles on phenotype. Several mutant alleles increase in frequency over GS evolution, and we 


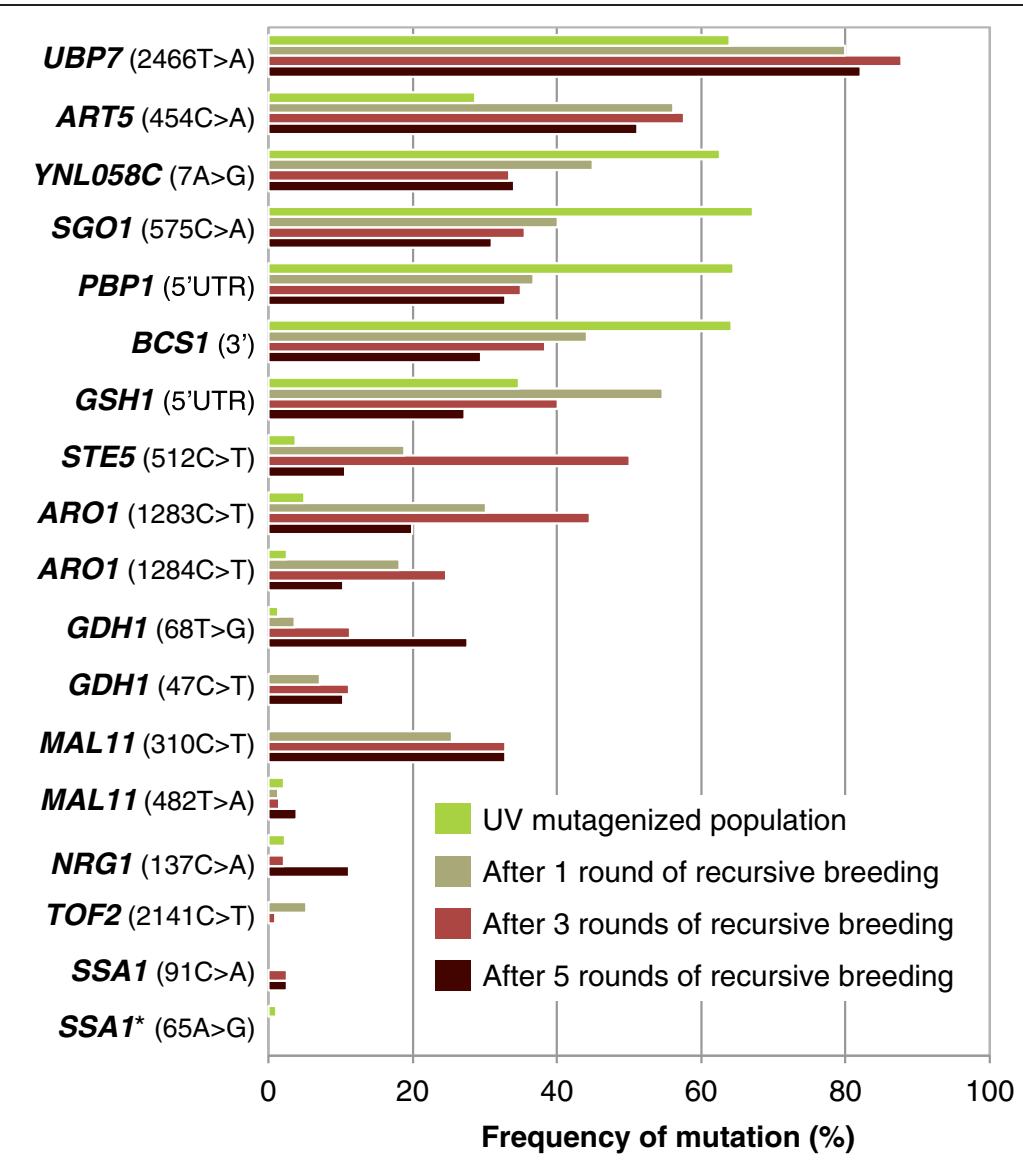

Figure 2 Frequency of R57 mutations throughout GS evolution. The haploid UV mutant population (UV), generated from a single round of UV mutagenesis, and rounds 1, 3 and 5 of GS evolution (R1, R3 and R5, respectively) were PCR amplified at the R57 mutation loci and deep sequenced to determine the relative frequency of sequence reads bearing mutation (above a threshold of 1\% of total sequence reads). The asterisk denotes mutations discovered that are not found in R57. UV, ultraviolet.

hypothesize that this increase is due to a beneficial phenotypic effect that is generated through GS evolution and repeated selection. These include the ART5, UBP7, SSA1, STE5, NRG1, MAL11 (leading to Mal11p - P104S) and both GDH1 mutations (Figure 2). This analysis also yields an additional SNP that falls within the area of PCR amplification, affecting SSA1 26 bp upstream of the R57 mutation and leading to a D22G missense mutation, which further supports a determinant role for SSA1 in the observed phenotype. The NOP58, STE5 (silent), DOP1, FIT3 3' UTR mutations were not located at a high enough density within the sequenced mutant populations to surpass our detection threshold.

Five mutations (located upstream of $B C S 1$ and $P B P 1$ and in the ORFs of YNL058C, SGO1 and UBP7) are represented at approximately $60 \%$ frequency in the UV mutant population. Due to the virtually identical representation of these five mutations within the UV mutant population, we hypothesized that they may arise from a single, highly tolerant haploid strain. The mutated loci identified in R57 were sequenced from seven random, discernible colonies selected from the frontier of growth for the UV mutant populations on HWSSL gradient agar plates (Figure 1B). Each of the single colonies contained the BCS1, PBP1, YNL058C, SGO1 and UBP7 mutations, corroborating our hypothesis that a single mutant strain present after UV mutagenesis was likely enriched to represent a large portion of that population. Assuming this mutant strain harboured at least one particularly productive mutation, propagation of these alleles to strain R57 may be a likely outcome. Heterogeneous population sequencing shows that of these five mutations, only the UBP7 mutation increased in frequency through GS evolution while the other four decreased in frequency (Figure 2). We hypothesize that this finding is indicative of a set of four non-productive or less important mutations found with the productive UBP7 mutation within a single genome, and when meiotic recombination occurs, linkage to the productive mutation diminishes until it reaches a steady state within the evolving population.

The mutation in the 5' UTR of GSH1 was identified in approximately $30 \%$ of sequence reads generated for 
this locus (Figure 2) in the UV population and was also identified in each of the seven UV mutant isolates that contain BCS1, PBP1, YNL058C, SGO1 and UBP7 mutations. It is therefore likely that all six mutations arose in a single mutant strain. The reason for the discrepancy in allele frequency between GSH1 and the other five mutations from UV mutant population sequencing is unknown but may be a population sequencing artefact. Unlike BCS1, PBP1, YNL058C and SGO1, the 5' UTR GSH1 mutation increases in frequency in the first three rounds of evolution (Figure 2) and therefore, as with $U B P 7$, more likely contributes to the tolerance phenotype than the other four mutations.

Several mutations that comprise a large part of the first three rounds of GS, and are therefore likely playing a determinant role in HWSSL tolerance, decrease in population frequency in the fifth round of GS (UBP7, ART5, ARO1). We hypothesize this occurs due to competition from strains bearing mutations that were rare in the initial mutant pools (that is, NRG1 and GDH1) or strains harbouring rare recombination events of multiple mutations that have resulted in augmented fitness.

\section{Analysis of R57 SNPs in isolates of GS round 5 heterogeneous population}

To identify possible combinations or permutations of mutations enriched through evolution, 20 strains isolated from the growth frontier of the fifth round of recursive
GS (Figure 1B lane R5) were sequenced via the Sanger method at each of the mutation loci. All of the strains show heterozygosity in at least one of the mutation loci. The results show a heterogeneous population of mutations that are found together in one strain (Figure 3). Of these isolates, $\geq 70 \%$ contain at least one mutation in $U B P 7, A R T 5$ and either of the GDH1 mutation loci, which further supports determinant roles for these genes on the phenotype. Several of the strains contain very few of the R57 mutations, which may indicate that not all R57 mutations are needed for HWSSL tolerance and likely that other unidentified mutations present within the round 5 population contribute to the tolerance trait. The percentages of mutated alleles within mutant populations as enumerated by population sequencing and single colony sequencing are similar (Figure 4); discrepancies in these frequencies may be due to the relatively large difference in sample size. General trends as to allele frequency are easily apparent and support our ability to generalize allele frequency within GS mutant populations by sequencing PCR-amplified mutation loci. The GS population sequencing data support determinant roles in HWSSL tolerance for a large portion of the mutated R57 genes based on their pervasiveness throughout the evolving populations. However, the most highly enriched mutations that increase over the strain evolution are of particular interest for reverse engineering studies. These include $U B P 7$, GDH1, ART5, ARO1, STE5 and MAL11.

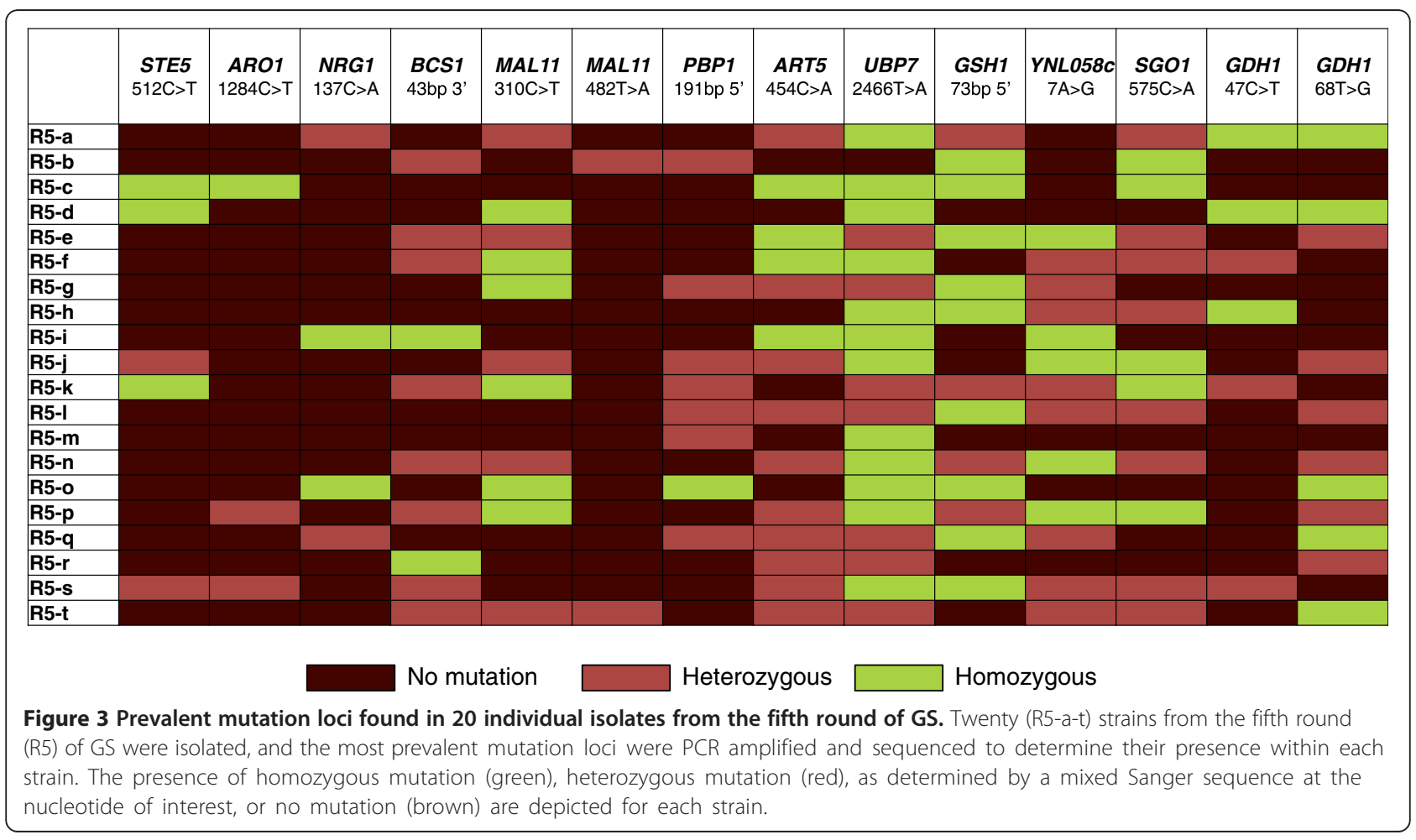




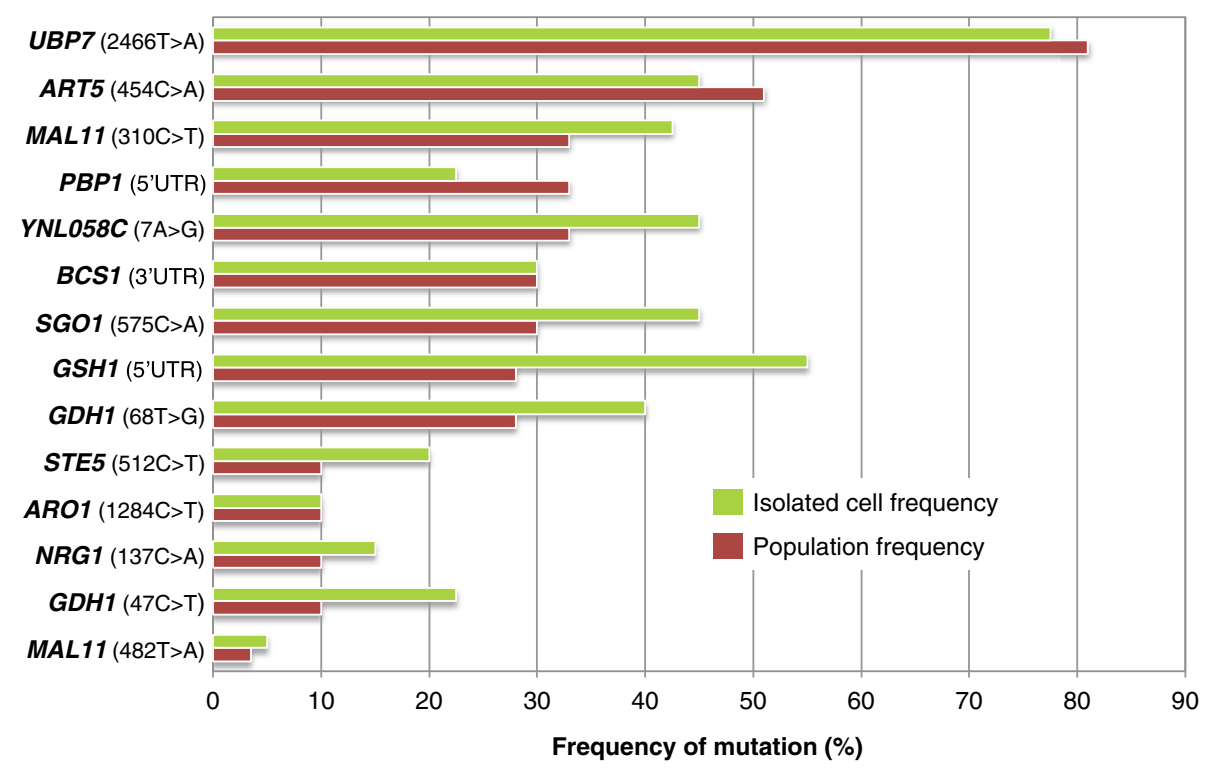

Figure 4 Comparison of mutation frequencies between population and single colony at mutation loci. Twenty single colonies were sequenced using the Sanger method at the most prevalent R57 mutation loci for comparison to deep sequencing results of mutation loci within the entire GS-evolved population.

\section{RNA-seq gene expression analysis}

To measure the impact of the mutations on gene expression in strain R57 and to probe for biological processes related to HWSSL tolerance, the gene transcription profile of strain R57 was compared to the WT diploid under control conditions (growth in defined medium, see 'Materials and methods') (Additional file 4: Data S2). Functional clustering was performed on the differentially expressed genes to discover enriched functional roles of gene products and biological pathways of interest (Figure 5).

These analyses identified 149 differentially expressed genes (>2-fold) (Additional file 3: Figure S3 and Additional file 4: Data S2). None of the 16 genes harbouring a mutation (Table 1) are found in this group with the exception of NRG1, which is upregulated 3-fold. Clustering of the 131 upregulated R57 genes as compared to the WT includes the major cluster of translation-related genes, mainly associated with ribosome biogenesis and translation regulation and 15 genes related to monosaccharide metabolism. These findings suggest a more active metabolism of R57 in early stationary phase, which may be related to growth differences between the WT and R57 [8]. Indeed, R57 displays a similar growth rate to the WT but reaches a lower optical density (OD) at stationary phase under non-inhibitory conditions with residual glucose remaining in the R57 medium (Additional file 3: Figure S4). The remaining upregulated enrichment clusters include genes related to cell wall organization, the cell membrane, ubiquitin-like (UBL) conjugation and organic acid synthesis pathways. Only 18 genes were downregulated under non-inhibitory conditions, resulting in 3 clusters of genes that are highly enriched (Figure 5). These include genes related to NADH/alcohol metabolism and metal-ion metabolism or are associated with cellular membrane transport or lipid metabolism.

UBP7 and the ubiquitin-mediated protein homeostasis machinery are determinants of HWSSL tolerance

$U B P 7$ bears a mutation that gains in frequency to represent a large portion of the GS-evolved population (Figure 2). Functional analysis suggests highly probable effects on protein structure and function due to this mutation, and $U B P 7$ is known to have a high degree of interaction with mutated R57 genes (Table 1, Additional file 2: Methods, Additional file 3: Figures S1 and S2). The RNA-seq data also shows enrichment clustering for increased expression of genes that encode proteins related to the ubiquitin-mediated proteolytic machinery (UBL conjugation) (Figure 5). However, the genes within these UBLconjugated gene clusters are associated with diverse biological processes, many of which do not play a direct role in the ubiquitination of proteins or ubiquitin-mediated proteolysis. Two notable exceptions, $U B I 2$ and $U B I 3$, which show increased expression in R57 relative to the WT (2.7- and 2.8-fold, respectively; Additional file 4: Data S2), encode ubiquitin fused to ribosomal proteins [31] and are responsible for generating ubiquitin as a fusion protein that is then cleaved to yield free ubiquitin by deubiquitinases. Most of the UBL conjugation cluster genes encode proteins that are regulated by this mechanism. Many of these genes are stress-tolerance related 


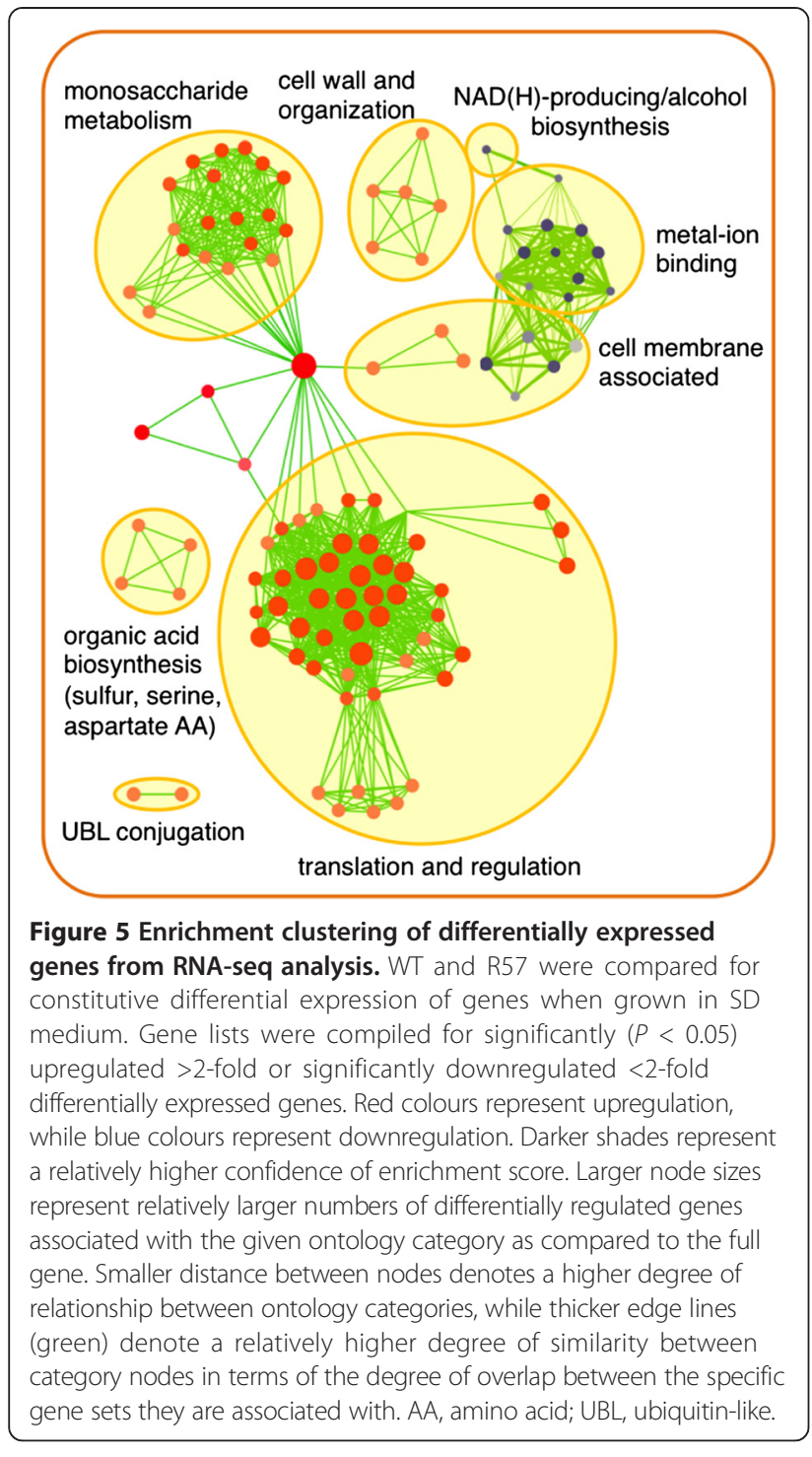

(RHR2, PUN1, ENA5, PDR5, PDR12). This suggests that stress tolerance genes showing increased expression due to HWSSL exposure may be also differentially controlled at the protein level by a modified ubiquitination machinery. Altogether, a significant portion of the HWSSL tolerance trait shown by R57 seems to be a direct result of changes in ubiquitin-mediated proteolytic pathways.

Protein damage and aggregation are likely a source of toxicity in cells exposed to lignocellulosic hydrolysates and have at least been partially shown to arise due to ROS damage from furan aldehyde exposure [32]. Cells regulate protein quality through destruction of misfolded or damaged polypeptides largely through selective, energy-dependent labelling with ubiquitin leading to digestion by the $26 \mathrm{~S}$ proteasome complex [33]. UBP7 encodes a ubiquitinspecific protease that cleaves ubiquitin-protein fusions [34], and as such, it is part of this ubiquitin-induced signalling machinery of the cell [35-37]. The cell's requirement for available ubiquitin increases during stress exposure [38]. Deubiquitinating enzymes act to recover ubiquitin from ubiquitin-protein conjugates and may therefore have a direct bearing on cellular protein and ubiquitin homeostasis [37]. It has already been shown that mutations within a deubiquitinase enzyme, $U B P 6$, can dramatically change steady-state ubiquitin levels within a cell [39], which is known to affect tolerance to a variety of stressors [40-42] and yeast prion toxicity [42]. Furthermore, upregulation of $U B P 13$, another yeast deubiquitinating enzyme, is beneficial to cells under cold stress and suggests that altering ubiquitin-induced signalling may be a viable path towards other forms of stress tolerance [43].

In order to test the role of the UBP7 $2466 \mathrm{~T}>\mathrm{A} \mathrm{mu}$ tation in hydrolysate tolerance, we replaced both WT copies of $U B P 7$ with this gene variant in a diploid WT CEN.PK background. The homozygous UBP7 $2466 \mathrm{~T}>\mathrm{A}$ strain was able to colonize a higher concentration of HWSSL on gradient agar plate screening (Figure 6) compared to the WT, but its tolerance to HWSSL is still below that of R57. The phenotype conferred by the UBP7 mutant does not reconstitute the full HWSSL tolerance displayed by R57 and supports the hypothesis that the high level of tolerance shown by R57 is a result of several mutations incorporated through GS.

One role of ubiquitination is the internalization of cell surface proteins [44-46]. This function relates to Art5p, which belongs to the ART (arrestin-related trafficking) family of proteins that are believed to function as adaptors for Rsp5p, a ubiquitin ligase that promotes endocytosis of plasma membrane proteins, including transporters, targeting damaged or unneeded plasma membrane proteins for vacuolar degradation [47]. Mutation of ART5 may represent a way for R57 to regulate destruction of proteins damaged by HWSSL stress or direct changes to the plasma membrane in order to respond more efficiently to the toxic HWSSL environment. As might be expected of a leucine to isoleucine mutation, like that found in the R57 Art5p L152I protein, this change is expected to be tolerated by Art5p (Table 1). Nevertheless, the high and increasing frequency of the ART5 454C > A mutation shown in the mutant pool sequencing experiment (Figure 2), the differential regulation of cell surface remodelling genes between the WT and R57 (Additional file 4: Data S2) and the proven role of ubiquitin-mediated degradation-machinery gene UBP7 in HWSSL tolerance suggest that this mutation might also play a determinant role in HWSSL tolerance.

\section{Nrg1p as a determinant in the inhibitor tolerance trait}

As the sole transcription factor-encoding gene located amongst R57 mutations, the NRG1 137C > A may result in the most pervasive phenotypic consequences. The homozygous P46Q mutation of Nrg1p was predicted to 


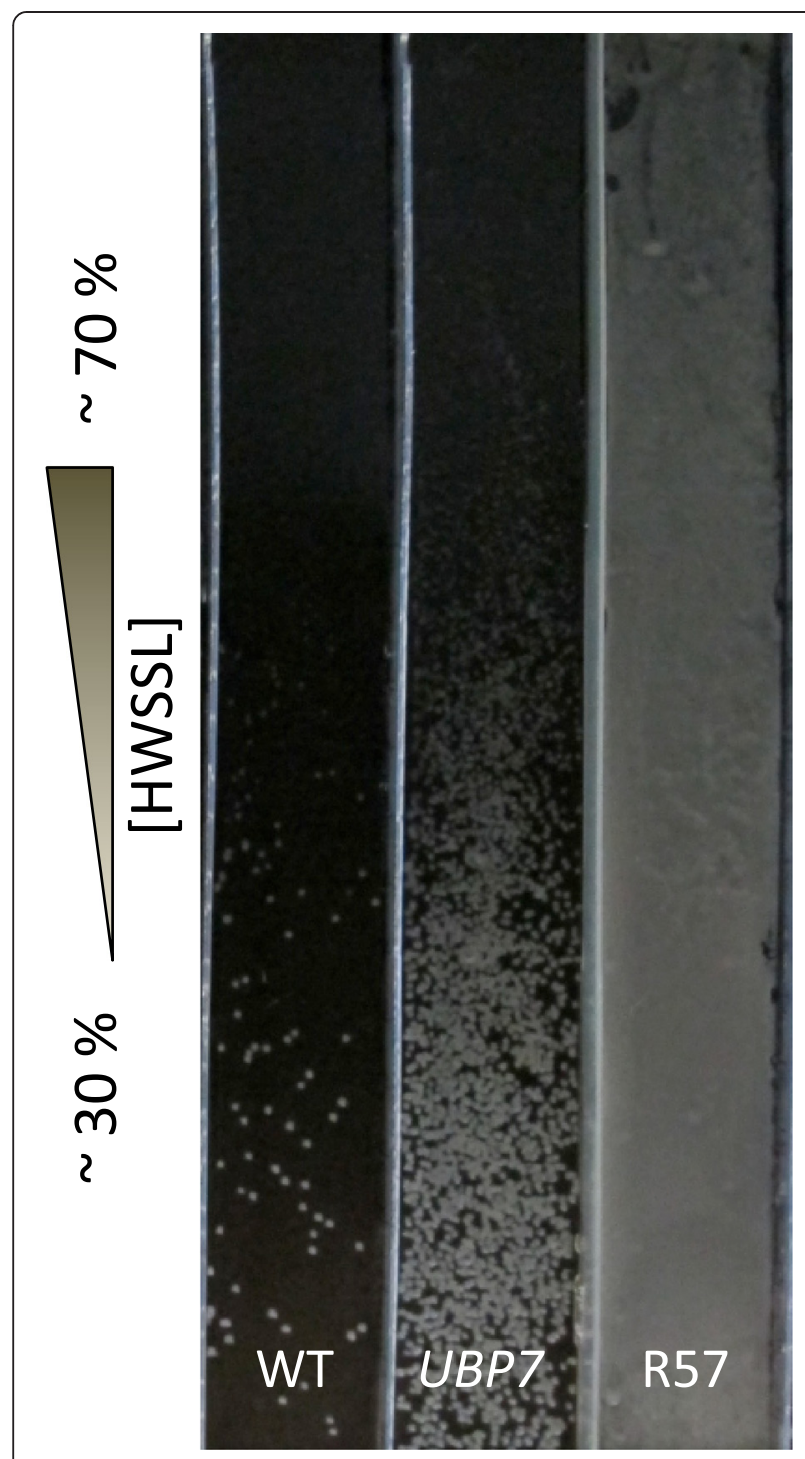

Figure 6 Testing of UBP7 mutation using HWSSL gradient agar plate screening. A HWSSL gradient agar plate (approximately 30\% to $70 \%$ HWSSL from bottom to top of plate) was spread with cells from cultures of (from left to right lanes) CEN.PK 113-7D diploid (WT), CEN.PK 113-7D diploid bearing the homozygous UBP7 mutation from R57 (UBP7), and strain R57 (R57). HWSSL, hardwood spent sulphite liquor; $\mathrm{WT}$, wild-type.

be non-tolerated with a SIFT score of 0.00 (Table 1), as proline is strictly conserved at this position and its substitution would result in altered protein function. SNP analysis of the GS-evolved populations showed a diminished prevalence of this mutation after mating of the UV-treated haploid population (Figure 2), leading to the hypothesis that the NRG1 mutation results in a recessive, loss-of-function mutation. The NRG1 137C > A allele gains prevalence in the GS populations after that point, present at a frequency of approximately $2 \%$ after three rounds and approximately 11\% after five rounds of pool-wise recursive breeding, when two copies of the allele are more likely present in single strains.

Nrg1p recruits the Tup1p-Cyc8p complex to repress gene expression. Therefore, the Nrg1p P46Q mutation may decrease repression of Nrg1p-controlled genes. NRG1, which self-regulates its transcription [48-51], was upregulated 3-fold in R57 over WT (Additional file 4: Data S2). The closely related transcription factor NRG2 was similarly upregulated approximately 5-fold in R57 over WT. In addition, four of the five most highly upregulated genes in R57 are known to be regulated by Nrg1p. These genes include CWP1 (approximately 13-fold upregulated), YLR015C (approximately 44-fold upregulated), GAT3 (approximately 23-fold upregulated) and TDA6 (approximately 14-fold upregulated) (Additional file 4: Data S2). In S. cerevisiae, the most significant transcriptional responses governed by $\mathrm{Nrg} 1 \mathrm{p}$ are all related to a multitude of stress conditions [52]. One of the main functional gene categories controlled by Nrg1p is related to peroxide tolerance, which is a specific trait of R57 [8]. Additionally, Vyas et al. reported that Nrg1-2p regulates a set of stress response genes and $\Delta n r g 1 \Delta n r g 2$ deletion mutants exhibit tolerance to oxidative stress and salt exposure [53], a trait that is also shared by R57 [8].

R57 also displays acetic acid tolerance after pre-exposure to HWSSL [8]. Nrg1p can directly repress genes that are activated by the downstream action of protein kinase Snf1p [54], which stimulates upregulation of stress responsive genes [55] and the transcription activator Haa1p, which imparts acetic acid tolerance [56]. Many of the genes highlighted as members of enriched clusters that show increased expression in $\mathrm{R} 57$ are known constituents of the Haa1p regulon including AQR1, HSP26, MSN4, PDR12, PDR16, SPI1, SUR2, SSE2, TDA6, TPO1, TPO2 and TPO3 (Additional file 4: Data S2). Genes like Haa1pdependent TPO2 and TPO3 (Nrg1p-regulated [49]) show a prominent effect on acetic acid tolerance [57]. Overall, our data support a strong role for Nrg1p in the control of hydrolysate inhibitor tolerance.

\section{A determinant role for GDH1 in HWSSL tolerance}

The presence of mutations predicted as non-tolerated in both copies of GDH1 and their close proximity (Table 1) strongly suggest a determinant role for GDH1 in HWSSL tolerance. Furthermore, population sequencing shows a steady increase in mutant allele frequency at both loci (Figure 2). GDH1 encodes an NAD(P)H-dependent glutamate dehydrogenase that catalyses the reductive amination of $\alpha$-ketoglutarate to yield glutamate, responsible for the majority of cellular nitrogen in S. cerevisiae via ammonium assimilation [58]. GDH1 is recognized as a determinant of resistance to acetic acid $[59,60]$. Likewise, recent proteomics studies using a strain that is tolerant of furfural, phenol and acetic acid show a downregulated 
nitrogen assimilation machinery, including Gdh1p [59]. It is believed this occurs in order to slow growth and allow stress tolerance mechanisms to protect the cell more effectively. A decrease in biomass yield exhibited by $\mathrm{R} 57$ relative to the WT under permissive conditions (Additional file 3: Figure S4) suggests that metabolism has shifted to a state of decreased resource utilization efficiency. As GDH1 is a central hub of nitrogen metabolism, microbial substrate tolerance engineering studies that focus on this gene are warranted, especially on the HWSSL substrate that was used to evolve strain R57, which is generally high in ammonia content (approximately $1 \% \mathrm{w} / \mathrm{v}$ [22]) and could lead to ammonia toxicity [61]. Bayer et al. recently showed that by increasing expression variability of GDH1 alone, one can tune the metabolism of a cell so that it responds more efficiently to limiting or toxic levels of ammonia [62].

The NADPH cofactor requirement of GDH1 is also a major consideration in attempting to explain the consequences of the GDH1 mutations. Yeast detoxifies the furan aldehyde inhibitors found in lignocellulosic hydrolysates by way of NADH/NADPH requiring enzymes [13]. Differential gene expression analysis between the WT and R57 show increased expression of GRE3 (Additional file 4: Data S2) in R57, encoding for a methylglyoxal reductase that can reduce furan aldehyde inhibitors via NADH [14]. The NAD(P)H cofactor usage of R57 may be modulated by a modified GDH1p, providing the reducing equivalents needed to detoxify the HWSSL furan aldehyde inhibitors.

\section{Sequencing supports determinant HWSSL tolerance roles for mutated genes SSA1, ARO1, MAL11 and $G S H 1$}

Given the GS methodology, it is likely that several of the accumulated mutations influence hydrolysate tolerance. This study was able to generate more restricted evidence that the SSA1, ARO1, MAL11 and GSH1 mutations found in R57 may be affecting HWSSL tolerance.

SSA1, also related to protein homeostasis, bears a mutation in R57. Ssa1p is a member of the heat shock 70 (Hsp70) family of proteins, which consists of highly conserved, broad specificity, essential protein chaperones (for reviews, see $[63,64])$. The tendency of harsh conditions to damage proteins and lead to aggregation [33] likely makes the role of Hsp-encoding genes important for HWSSL tolerance. The R57 Q31K mutation of Ssalp is located at a highly conserved residue, as predicted by SIFT (Table 1). Although not highly represented in population sequencing, the discovery of an adjacent SSA1 mutation (D22G) that is also present within the tolerant population (Figure 2) suggests that this region of Ssa1p may have bearing on the R57 phenotype. The structure of Ssalp contains two distinct domains, the nucleotide-binding domain (NBD) that is responsible for binding and hydrolyzing ATP and the substratebinding domain which can bind short hydrophobic segments of incompletely folded or unfolded polypeptides, in order to prevent adverse aggregation [63]. The Q31K mutation of Ssalp is located in the NBD, and when the Escherichia coli Hsp70 homolog DnaK is used as a structural reference, the mutation is shown to lie adjacent to the ATP-binding pocket [65]. Although the Q31K and D22G mutations in Ssa1p have not been studied thus far, residues in this area of the protein have been shown to influence its NBD function and folding activity $[65,66]$.

Furthermore, Arolp, which catalyses steps 2 to 6 of the chorismate pathway leading to synthesis of aromatic amino acids [67], harbours a predicted phenotype-conferring mutation that gains in frequency within sequenced mutant pools. After an acetic acid challenge, aromatic amino acid synthesis and tryptophan synthesis in particular are pathways that are found to be upregulated [60], while mutants auxotrophic for aromatic amino acid synthesis show acetic acid sensitivity [68] and deletion of ARO1 leads to sensitivity to osmotic and ethanol stress [69].

Mal11p bears two mutations that pervade and increase in the GS-evolved HWSSL-tolerant population. Mal11p is a trehalose- $\mathrm{H}^{+}$symporter $[70,71]$ and could be related to osmotic stress protection via trehalose transport or $\mathrm{pH}$ stasis due to its proton requirement. Although the effect of MAL11 on tolerance to industrial processes has not been demonstrated, it is a common trend for the genome of industrial yeast strains to show a loss or reduction of MAL11 genes [72-74].

Finally, the mutation in the 5' UTR of GSH1 may potentially be affecting redox homeostasis by influencing glutathione levels; GSH1 can influence tolerance to lignocellulosic hydrolysate inhibitors in this way [75]. Glutathione is comprised of glycine, cysteine and glutamate and is a major redox buffer of the cell, cycling between its reduced and oxidized form, relying on NADP $(\mathrm{H})$ for recycling. Hydrogen peroxide induces GSH1 transcription but relies on the presence of intercellular amino acid pools, namely glutamate, glutamine and lysine to induce glutathione production [76]. Therefore, modified glutamate assimilation via GDH1 mutation and the 5' UTR mutation in GSH1 both potentially affect intercellular glutamate pools and concomitant expression of GSH1. Between the WT and R57, genes that lead to cysteine biosynthesis (HOM2, MET16, MET17 and MET3), along with genes leading to glutamate and lysine biosynthesis (HOM3, LYS9 and LYS12), are upregulated (Additional file 4: Data S2). This finding suggests that R57 has upregulated pathways towards glutathione precursor generation as part of its physiology and constitutes a possible link to the GSH1 5' UTR mutation. 


\section{GS population sequencing provides evidence against prominent phenotypic roles of isolated mutations}

Although some of the remaining R57 mutations may be of interest based on the known functions of the affected genes, sequencing of GS populations did not support a determinant effect on HWSSL tolerance for every mutation. Namely, the mutations located in the $5^{\prime}$ UTR of PBP1, 3' of BCS1 and in SGO1 and YNL058C seem to be linked in a single mutant that comprised a large proportion of the UV mutant population, but decreased in frequency throughout GS evolution. The verified tolerance-conferring effect of the UBP7 mutation to which they were initially linked, and which gained in frequency over population evolution, suggests that these linked mutations were merely carried through to strain R57 from UV mutagenesis. The TOF2 mutation gained in frequency within the GS-evolved strains at round 1 but diminished in later rounds, while the DOP1 and FIT3 3' UTR mutations were not highly represented in the GS populations and may be relatively specific to R57, which devalues these genes as potential tolerance-associated determinants. One exception is the mutation in Ste5p, which increased in frequency throughout GS, suggesting that it may play a role in the evolution of R57. However, since Ste5p is involved in mating, which is essential to the GS method used to generate R57, a strain with a modified mating behaviour may play a role in GS evolution but is likely not linked to stress tolerance.

\section{Conclusions}

GS theory asserts that beneficial mutations accumulate within strains to rapidly evolve a trait of interest while simultaneously eliminating detrimental mutations. Our data suggest that during GS evolution, there is accumulation of complementary mutations in key cellular processes through recursive genetic recombination or by the accumulation of single mutations in crucial genes that confer a fitness advantage. Mutations that lead to a large fitness advantage, such as $U B P 7$, may become highly represented in the initial mutant pool and lead to over-representation of non-productive mutations found in the same strain through genetic linkage. The decrease in the frequency of these non-productive mutations during GS evolution suggests that mutations of lesser or no impact on the trait of interest can be crossed out of final strains.

As a workflow, meiotic recombination-mediated GS of $S$. cerevisiae, combined with genome resequencing, population sequencing of mutation loci and RNA-seq transcriptional profiling, generated complementary results that provided novel insights into tolerance to a specific lignocellulosic hydrolysate, along with gene targets that can be used for strain engineering. The assortment of processes and genes involved in inhibitor tolerance could not have been rationally determined prior to this study.
This study provides insights into the multiple biological processes that act in concert to establish tolerance to a multi-inhibitory substrate. Our strongest evidence supports determinant roles for Ubp7p and Art5p, related to ubiquitin-mediated proteolysis; stress response transcriptional repressor, Nrg1p; and NADPH-dependent glutamate dehydrogenase, Gdh1p. However, important roles in hydrolysate tolerance are supported for several of the mutations discovered in the GS-evolved strains and populations (SSA1, ARO1, MAL11 and GSH1), and the potential phenotypic impact of each mutation has not been ruled out. Therefore, a subsequent study is ongoing in our lab to examine the effect of each mutation discovered in R57 and explore potential epistatic effects between the mutations reported here. As whole genome sequencing becomes ever more accessible, and as the biotechnology industry requires biocatalysts with increasingly complex traits, GS followed by analyses like those carried out in this study stands to have a rapid and profound effect on our understanding of complex multigenic traits.

\section{Materials and methods}

\section{Strains and materials}

The S. cerevisiae CEN.PK strains, supplied by EUROSCARF (Institute for Molecular Biosciences, Frankfurt, Germany), were used as the WT reference and progenitor strains for genome-shuffled mutant populations, including prototrophic diploid strain CEN.PK 122 and haploid strains CEN. PK 113-1A (MAT $\boldsymbol{\alpha})$ and 113-7D (MAT a). The haploid CEN.PK strains were used in a previous study to generate HWSSL-tolerant strain R57.

The HWSSL used for all experiments was provided by Tembec Inc. (Temiscaming, Quebec, Canada). HWSSL was adjusted to pH 5.5 with $10 \mathrm{M} \mathrm{NaOH}$ and contained, on average $(w / v), 0.076 \%$ arabinose, $2 \%$ xylose, $0.16 \%$ galactose, $0.24 \%$ glucose, $0.43 \%$ mannose, $1 \%$ acetic acid, $0.18 \%$ furfural and $0.11 \%$ HMF.

\section{Sequencing of WT and R57 strains}

Genomic DNA of WT haploid CEN.PK113-7D and the CEN.PK-derived R57 diploid yeast strains were isolated from overnight cultures grown in YPD using the DNeasy Blood \& Tissue Kit (Qiagen, Toronto, Ontario, Canada). Library construction and sequencing were done at the Michael Smith Genome Sciences Centre using an Illumina $1 G$ Genome Analyzer (Illumina, Inc., San Diego, CA, USA). Two lanes were sequenced for the WT strain and four for strain R57. All genome sequence data and RNA-seq read data from this publication have been submitted to the National Center for Biotechnology Information (NCBI) sequence read archive under BioProject \# PRJNA231093. 


\section{Sequencing alignment and mutation calling}

Sequencing reads were aligned to the WT CEN.PK113-7D reference genome obtained from the NCBI (PRJNA52955) [77] and cross-referenced with read alignments obtained in our laboratory using our WT consensus sequence that was created using the S288c genome sequence as an alignment backbone. Alignments were performed both with Bowtie using standard parameters [78] and CLC Genomics Workbench version 5.1 with default parameters and ignoring non-specific matches. SNP and indel calling were both performed with Maq version 0.7.1 [79] and CLC Genomics Workbench for verification and visualization. To eliminate false positives in mutation calling, the DNA sequencing reads obtained from the WT were subjected to the same variation calling protocol as strain R57. Variations that were called when CEN.PK113-7D Illumina reads were aligned onto the CEN.PK113-7D consensus genome sequence and those that corresponded to variations called for R57 reads aligned onto the CEN. PK113-7D consensus sequence were discarded as false positives. False positive SNP calls were likely derived from sequence-specific miscall errors [80] or due to alignments in non-specific regions of the genome or to areas of low complexity [81]. The coverage requirement for SNP calling for WT reads was also lowered to $\leq 5$-fold in order to ensure that SNPs that may result due to misalignment could be easily identified. These miscalls were edited out of the final mutation list by manual inspection and visualization with CLC Genomics Workbench. Maq SNP analysis was performed using the cns2snp command, and SNPs were called if the region upon which they were mapped returned a genome copy score $=1$ and carried $\mathrm{a}$ Phred-like quality score $\geq 40$. SNP analysis was corroborated with CLC Genomics Workbench SNP Detection function with a quality score of $\geq 40$ for the central base and $\geq 30$ for the surrounding bases. The threshold for variation at a specific base in the genome needed for SNP calling was lowered to $\geq 10 \%$ of reads, yielding an aberrant base call for the CEN.PK113-7D read alignment from $\geq 35 \%$ for R57 SNP calling in order to maintain stringency on positive variation calling. Therefore, if a SNP was called for more than $35 \%$ of the reads in $\mathrm{R} 57$ but was also called for $10 \%$ or more of the reads for the WT control, it was discarded as a false SNP call. Indel analysis was also performed with Maq using the Indelpe command and verified with CLC Genomics Workbench, both under standard parameters and compared to WT reads for control, as described above. Copy number variation was assessed using CNV-seq [82] to compare WT to R57 reads with a log2 threshold of 0.75 , below the level used to detect reliable CNVs in CEN.PK [77]. The mutations identified were verified by Sanger sequencing and compared to the other Mat parental WT, CEN.PK113-1A, to ensure the mutations were not present in either of the WT parental haploid strains. Locations of mutations were assessed using the CEN.PK consensus sequence as a guide.

\section{Protein impact assessment of ORF-located mutations}

Mutations in ORFs were examined by translating DNA sequences using ExPASy translate [83] and performing a BLAST comparison using the BLAST2Seq software program hosted by NCBI. Mutational impact assessment was done with the SIFT program $[28,29]$ using recommended best practices [28]. Homologous proteins with $<90 \%$ identity were chosen for comparison of the degree of conservation of the amino acid position in question for up to 100 homologous proteins. Amino acid substitutions were predicted as leading to a phenotype if the SIFT score was $\leq 0.05$ and tolerated if the score is $>0.05$.

\section{Sequencing of R57 SNPs in GS heterogeneous populations}

The following heterogeneous populations of cells were used to track R57 SNP frequency through GS evolution: WT diploid CEN.PK122, a pooled UV mutagenized population (three pools of CEN.PK113-1A and two pools CEN. PK113-7D UV mutants), along with cells from the population of the first, third and fifth rounds of recursive GS obtained from our previous study that generated R57 [8]. Cells from each population were selected from above the frontier of WT growth as observed by screening on HWSSL gradient agar plates (Figure 1B), yielding populations that were enriched for tolerance to HWSSL, as described [8]. A sample of $10^{9}$ cells, suspended in phosphatebuffered saline (PBS), from each population was spread onto a single lane of the gradient plate and incubated for 6 days at $30^{\circ} \mathrm{C}$. Each plate contained two lanes spread with CEN.PK 122 cells for comparison to each individual GS population, in duplicate. Each plate was screened in biological duplicates.

Cells that grew to higher HWSSL concentrations than the WT on the gradient plates were scraped, suspended in PBS and adjusted to approximately $4 \times 10^{8}$ cells $/ \mathrm{mL}$ using a haemocytometer. For each population, DNA from approximately $4 \times 10^{7}$ cells was extracted using a DNeasy Blood \& Tissue Kit (Qiagen, Toronto, Ontario, Canada). Five microlitres of each genomic DNA preparation was used as template for PCR with primers specific for each of the 20 SNP regions, located at approximately $50 \mathrm{bp}$ from either end of the SNP. The primers were designed with sequencing adapter attachment for use with the Ion Torrent Personal Genome Machine (Life Technologies, Carlsbad, CA, USA) according to the manufacturer's instructions (Additional file 2: Table S1). All PCR products were gel purified and quantified in triplicate using the Promega Quantifluor dsDNA system (Promega Corporation, Madison, WI, USA). The PCR products were diluted to $16 \mathrm{pmol}$, and $20-\mu \mathrm{L}$ samples from each reaction were pooled to 
make four pools (one UV mutagenized and three GS pools). Pools of PCR products were sequenced using a 316 chip with the 200-bp kit (following the Ion Torrent protocols).

\section{Sequencing SNPs from isolates of the round 5 GS heterogeneous population}

Twenty isolated colonies picked from the growth frontier of a HWSSL gradient plate of the round 5 GS population were streak purified on 50\% $(v / v)$ HWSSL and 2\% agar $(w / v)$ Petri plates. Colonies isolated from the plates were grown overnight in $5 \mathrm{~mL}$ YPD broth at $30^{\circ} \mathrm{C}$, and DNA was extracted with a DNeasy Blood \& Tissue Kit for use as a template in the PCR amplification of the mutated gene region. Primers used for amplification are described in Additional file 2: Table S2, and PCR products were sequenced using the Sanger method.

\section{Transcriptome analysis}

WT diploid strain CEN.PK122 and HWSSL-tolerant R57 were used in RNA-seq experiments. The WT and R57 strains were grown in $50 \mathrm{~mL}$ synthetic defined (SD) medium (yeast nitrogen base without amino acids $0.17 \%$ $w / v$, ammonium sulphate $0.5 \% w / v$, glucose $2 \% w / v$ ) overnight at $30^{\circ} \mathrm{C}$ under semi-fermentative conditions (sealed $125-\mathrm{mL}$ flasks shaken at $100 \mathrm{rpm}$ ) to early stationary phase (Additional file 3: Figure S4). Cultures were normalized to an $\mathrm{OD}_{600} \mathrm{~nm}$ of 3 , and two independently grown cell samples of these cultures were used for RNA extraction. For each sample, cells from $5 \mathrm{~mL}$ of culture were harvested by centrifugation at $1,800 \times g$ and $4^{\circ} \mathrm{C}$ and frozen in liquid $\mathrm{N}_{2}$ until RNA isolation. RNA extracts were prepared using the RNeasy Plant Mini Kit (Qiagen) according to manufacturer's specifications for use with yeast, in which frozen cells were suspended in lysis buffer and disrupted with a mini bead beater (Precellys 24, Bertin Technologies, Montigny-le-Bretonneux, France) at $4^{\circ} \mathrm{C}$. Prior to sequencing, RNA quality was confirmed using an Agilent 2100 Bioanalyzer (Agilent Technologies, Santa Clara, CA, USA).

RNA sequencing was performed at the McGill/Genome Quebec Innovation Centre in duplicate on the Illumina Genome Analyzer IIx and Illumina HiSeq 2000 for the WT vs. R57. DNA libraries were subjected to 36 or $50 \mathrm{cy}-$ cles of sequencing on the Illumina Genome Analyzer IIx and Illumina HiSeq 2000, respectively.

RNA-seq differential transcription analysis and statistical comparisons were performed with CLC Genomics Workbench version 5.1. The cDNA sequence reads from RNA-seq were trimmed to remove Illumina sequencing adaptors as well as unreliable read ends, and alignments were performed using the CEN.PK113-7D genome sequence and associated GTF file [77] as the backbone for alignment mapping and quantitation. Significance values for differential expression were computed using Baggerly's test [84]. The samples were then FDR-corrected in order to eliminate non-productive leads from the expression results. Gene transcripts showing differential expression with a corrected $P$ value of $<0.05$ and a $>2$ fold increase were used for functional clustering and enrichment mapping of differentially expressed genes.

Functional annotation clustering was executed with DAVID Bioinformatics Resources 6.7 [85]. Clusters of up or down expressed genes with gene ontology (GO) term enrichment scores of $\geq 1.3$ (equivalent to a non-log scale value of 0.05 ) are reported, unless stated otherwise. Enrichment maps of ontology categories from clustering were generated with the Enrichment Map 1.2 software plug-in for Cytoscape 2.8 [86,87]. All functional annotations presented were derived from SGD [88] or the DAVID server unless otherwise referenced. Transcription factor binding analysis was done through the YEASTRACT database [89-91].

\section{Reconstitution of the UBP7 mutation in WT}

To determine if the UBP7 mutation was contributing to the HWSSL tolerance phenotype of R57 as predicted, the mutation was introduced into WT and the resulting strain was tested for growth on HWSSL. The WT allele was replaced in CEN.PK113-7D via homologous recombination of a DNA cassette containing the mutated $U B P 7$ sequence flanked by a kanamycin resistance marker. Sanger sequencing of the PCR-amplified region was used to confirm that the transformants harboured the mutation. Homozygous diploid strains of the WT and $U B P 7$ mutant were created by mating type switching using the YCp50::HO plasmid [92] and mating haploid strains of opposite mating type. The UBP7 homozygous diploid mutant and WT diploid strains along with R57 were tested for their tolerance to HWSSL in parallel, as previously described [8].

\section{Additional files}

Additional file 1: Data S1. A summary mapping report for CEN.PK 113-7D.

Additional file 2: Supplemental methods. A document on assessing functional interaction of mutations. (DOCX $31 \mathrm{~kb}$ )

Additional file 3: Supplemental figures. Figure S1. Interaction map of genes affected by mutation in R57. Figure S2. Ontology categories associated with R57 genes affected by mutation. Figure S3. Differentially expressed genes comprising enrichment clusters based on biological function between the WT and R57. Figure S4. Cell growth and glucose consumption of WT and R57 mutant strains.

Additional file 4: Data S2. RNA-seq results for differentially expressed genes between WT vs R57 without HWSSL exposure.

\section{Abbreviations}

CNV: copy number variation; GS: genome shuffling; HMF: 5-(hydroxymethyl)2-furaldehyde; HWSSL: hardwood spent sulphite liquor; indel: insertion/ deletion; NBD: nucleotide-binding domain; UBL: ubiquitin-like. 


\section{Competing interests}

The authors declare that they have no competing interests.

\section{Authors' contributions}

DP and VM conceived and designed the study. DP, DC and $\mathrm{HJ}$ acquired the data. DP, DC and VM analysed and interpreted the data. Drafting of the manuscript was done by DP and DC. Critical revision of the manuscript for intellectual content was performed by DP, HL and VM. VM and HL obtained funding. All authors have read and approved of the final version of this manuscript.

\section{Acknowledgements}

An NSERC Strategic Project (GHGPJ322381), the NSERC Bioconversion Network (NETGP350246-07), the AAFC Agricultural Bioproducts Innovation Program (ABTP_000159), BioFuelNet and a Canada Research Chair to V.J.J.M supported this research. Dominic Pinel was supported by a graduate scholarship from Le Fonds Québécois de la Récherche sur la Nature et les Technologies.

\section{Author details \\ ${ }^{1}$ Department of Biology, Centre for Structural and Functional Genomics, Concordia University, 7141 Sherbrooke Street West, Montréal, Québec H4B 1R6, Canada. ${ }^{2}$ School of Environmental Sciences, University of Guelph, Guelph, Ontario N1G 2 W1, Canada. ${ }^{3}$ Current address: Energy Biosciences Institute, University of California, Berkeley, Berkeley, CA 94704, USA. ${ }^{4}$ Current address: Crabtree Nutrition Laboratories, McGill University Health Center, Montreal, Quebec H3A 1A1, Canada.}

\section{Received: 28 November 2014 Accepted: 17 March 2015 Published online: 31 March 2015}

\section{References}

1. Oud B, van Maris AJ, Daran JM, Pronk JT. Genome-wide analytical approaches for reverse metabolic engineering of industrially relevant phenotypes in yeast. FEMS Yeast Res. 2012;12:183-96.

2. Smith DR, Quinlan AR, Peckham HE, Makowsky K, Tao W, Woolf B, et al. Rapid whole-genome mutational profiling using next-generation sequencing technologies. Genome Res. 2008;18:1638-42.

3. Le Crom S, Schackwitz W, Pennacchio L, Magnuson JK, Culley DE, Collett JR, et al. Tracking the roots of cellulase hyperproduction by the fungus Trichoderma reesei using massively parallel DNA sequencing. Proc Natl Acad Sci U S A. 2009;106:16151-6.

4. Sarin S, Bertrand V, Bigelow H, Boyanov A, Doitsidou M, Poole RJ, et al. Analysis of multiple ethyl methanesulfonate-mutagenized Caenorhabditis elegans strains by whole-genome sequencing. Genetics. 2010;185:417-30.

5. Harper MA, Chen Z, Toy T, Machado IM, Nelson SF, Liao JC, et al. Phenotype sequencing: identifying the genes that cause a phenotype directly from pooled sequencing of independent mutants. PLoS One. 2011;6:e16517.

6. Parts L, Cubillos FA, Warringer J, Jain K, Salinas F, Bumpstead SJ, et al. Revealing the genetic structure of a trait by sequencing a population under selection. Genome Res. 2011;21:1131-8.

7. Zhang YX, Perry K, Vinci VA, Powell K, Stemmer WPC, del Cardayre SB. Genome shuffling leads to rapid phenotypic improvement in bacteria. Nature. 2002;415:644-6.

8. Pinel D, D'Aoust F, del Cardayre SB, Bajwa PK, Lee H, Martin VJJ. Saccharomyces cerevisiae genome shuffling through recursive population mating leads to improved tolerance to spent sulfite liquor. Appl Environ Microbiol. 2011;77:4736-43.

9. Patnaik R, Louie S, Gavrilovic V, Perry K, Stemmer WPC, Ryan CM, et al. Genome shuffling of Lactobacillus for improved acid tolerance. Nat Biotechnol. 2002;20:707-12.

10. Dai MH, Copley SD. Genome shuffling improves degradation of the anthropogenic pesticide pentachlorophenol by Sphingobium chlorophenolicum ATCC 39723. Appl Environ Microbiol. 2004;70:2391-7.

11. Biot-Pelletier D, Martin VJJ. Evolutionary engineering by genome shuffling. Appl Microbiol Biotechnol. 2014;98(9):3877-87.

12. Almeida JRM, Modig T, Petersson A, Hahn-Hägerdal B, Lidén G, Gorwa-Grauslund MF. Increased tolerance and conversion of inhibitors in lignocellulosic hydrolysates by Saccharomyces cerevisiae. J Chem Technol Biotechnol. 2007;82:340-9.
13. Liu ZL. Molecular mechanisms of yeast tolerance and in situ detoxification of lignocellulose hydrolysates. Appl Microbiol Biotechnol. 2011;90:809-25.

14. Liu ZL, Blaschek HP. Lignocellulosic biomass conversion to ethanol by Saccharomyces. In: Vertes A, Qureshi N, Yukawa H, Blaschek $H$, editors. Biomass to biofuels: strategies for global industries. West Sussex, U. K: John Wiley \& Sons, Ltd; 2010. p. 17-36.

15. Palmqvist $\mathrm{E}, \mathrm{Hahn}$-Hägerdal B. Fermentation of lignocellulosic hydrolysates. I: Inhibition and detoxification. Biores Technol. 2000;74:17-24.

16. Richardson TL, Harner NK, Bajwa PK, Trevors JT, Lee H. Approaches to deal with toxic inhibitors during fermentation of lignocellulosic substrates. Acs Sym Ser. 2011;1067:171-202.

17. Pinel D, Gawand P, Mahadevan R, Martin VJJ. 'Omics' technologies and systems biology for engineering Saccharomyces cerevisiae strains for lignocellulosic bioethanol production. Biofuels. 2011;2:659-75.

18. Gorsich SW, Slininger PJ, Liu ZL. Physiological responses to furfural and HMF and the link to other stress pathways. J Biotechnol. 2005;118:S91-1

19. Petersson A, Almeida JRM, Modig T, Karhumaa K, Hahn-Hägerdal B, Gorwa-Grauslund MF, et al. A 5-hydroxymethyl furfural reducing enzyme encoded by the Saccharomyces cerevisiae ADH6 gene conveys HMF tolerance. Yeast. 2006:23:455-64.

20. Keating JD, Panganiban C, Mansfield SD. Tolerance and adaptation of ethanologenic yeasts to lignocellulosic inhibitory compounds. Biotechnol Bioeng. 2006;93:1196-206.

21. Pinel D, Martin VJJ. Meiotic recombination-based genome shuffling of Saccharomyces cerevisiae and Schefferomyces stiptis for increased inhibitor tolerance to lignocellulosic substrate toxicity. In: Patnaik R, editor. Engineering complex phenotypes in industrial strains. 1st ed. Hoboken, New Jersey: John Wiley \& Sons, Inc; 2012. p. 233-50.

22. Helle SS, Murray A, Lam J, Cameron DR, Duff SJ. Xylose fermentation by genetically modified Saccharomyces cerevisiae 259ST in spent sulfite liquor. Bioresour Technol. 2004;92:163-71.

23. Olsson L, HahnHagerdal B. Fermentation of lignocellulosic hydrolysates for ethanol production. Enzyme Microb Tech. 1996;18:312-31.

24. Parajó JC, Domínques H, Domínquez JM. Biotechnological production of xylitol. Part 3: operation in culture media made from lignocellulose hydrolysates. Bioresour Technol. 1998;66:25-40.

25. Helle S, Duff S. Supplementing spent sulfite liquor with a lignocellulosic hydrolysate to increase pentose/hexose co-fermentation efficiency and ethanol yield. Final report-Natural Resources Canada-Tembec Industries; 2004. http://www.lifesciencesbc.ca/files/dufffinal_report.pdf.

26. Yassour M, Kaplan T, Fraser HB, Levin JZ, Pfiffner J, Adiconis X, et al. Ab initio construction of a eukaryotic transcriptome by massively parallel mRNA sequencing. Proc Natl Acad Sci U S A. 2009;106:3264-9.

27. Nagalakshmi U, Wang Z, Waern K, Shou C, Raha D, Gerstein M, et al. The transcriptional landscape of the yeast genome defined by RNA sequencing. Science. 2008;320:1344-9.

28. Kumar P, Henikoff S, Ng PC. Predicting the effects of coding non-synonymous variants on protein function using the SIFT algorithm. Nat Protoc. 2009:4:1073-81.

29. Ng PC, Henikoff S. SIFT: predicting amino acid changes that affect protein function. Nucleic Acids Res. 2003;31:3812-4.

30. Sim NL, Kumar P, Hu J, Henikoff S, Schneider G, Ng PC. SIFT web server: predicting effects of amino acid substitutions on proteins. Nucleic Acids Res. 2012;40:W452-7.

31. Ozkaynak E, Finley D, Solomon MJ, Varshavsky A. The yeast ubiquitin genes: a family of natural gene fusions. EMBO J. 1987;6:1429-39.

32. Modig T, Lidén G, Taherzadeh MJ. Inhibition effects of furfural on alcoho dehydrogenase, aldehyde dehydrogenase and pyruvate dehydrogenase. Biochem J. 2002;363:769-76.

33. Goldberg AL. Protein degradation and protection against misfolded or damaged proteins. Nature. 2003;426:895-9.

34. Hochstrasser M. Ubiquitin-dependent protein degradation. Annu Rev Genet. 1996;30:405-39.

35. Kimura Y, Tanaka K. Regulatory mechanisms involved in the control of ubiquitin homeostasis. J Biochem. 2010;147:793-8.

36. Hershko A, Ciechanover A. The ubiquitin system. Annu Rev Biochem. 1998;67:425-79.

37. Mukhopadhyay D, Riezman H. Proteasome-independent functions of ubiquitin in endocytosis and signaling. Science. 2007;315:201-5. 
38. Finley D, Ozkaynak E, Varshavsky A. The yeast polyubiquitin gene is essential for resistance to high temperatures, starvation, and other stresses. Cell. 1987;48:1035-46.

39. Hanna J, Meides A, Zhang DP, Finley D. A ubiquitin stress response induces altered proteasome composition. Cell. 2007;129:747-59.

40. Leggett DS, Hanna J, Borodovsky A, Crosas B, Schmidt M, Baker RT, et al. Multiple associated proteins regulate proteasome structure and function. Mol Cell. 2002;10:495-507.

41. Hanna J, Leggett DS, Finley D. Ubiquitin depletion as a key mediator of toxicity by translational inhibitors. Mol Cell Biol. 2003;23:9251-61.

42. Chernova TA, Allen KD, Wesoloski LM, Shanks JR, Chernoff YO, Wilkinson KD. Pleiotropic effects of Ubp6 loss on drug sensitivities and yeast prion are due to depletion of the free ubiquitin pool. J Biol Chem. 2003;278:52102-15.

43. Hernández-López MJ, Garcia-Marqués S, Randez-Gil F, Prieto JA. Multicopy suppression screening of Saccharomyces cerevisiae identifies the ubiquitination machinery as a main target for improving growth at low temperatures. Appl Environ Microbiol. 2011;77:7517-25.

44. Kolling R, Hollenberg CP. The ABC-transporter Ste6 accumulates in the plasma membrane in a ubiquitinated form in endocytosis mutants. EMBO J. 1994;13:3261-71

45. Hein C, Springael JY, Volland C, Haguenauer-Tsapis R, Andre B. NPI1, an essential yeast gene involved in induced degradation of Gap1 and Fur4 permeases, encodes the Rsp5 ubiquitin-protein ligase. Mol Microbiol. 1995;18:77-87.

46. Hicke L, Riezman H. Ubiquitination of a yeast plasma membrane receptor signals its ligand-stimulated endocytosis. Cell. 1996;84:277-87.

47. Lin CH, MacGurn JA, Chu T, Stefan CJ, Emr SD. Arrestin-related ubiquitin-ligase adaptors regulate endocytosis and protein turnover at the cell surface. Cell. 2008;135:714-25.

48. Goh WS, Orlov Y, Li J, Clarke ND. Blurring of high-resolution data shows that the effect of intrinsic nucleosome occupancy on transcription factor binding is mostly regional, not local. PLoS Comput Biol. 2010;6:e1000649.

49. Harbison CT, Gordon DB, Lee TI, Rinaldi NJ, Macisaac KD, Danford TW, et al. Transcriptional regulatory code of a eukaryotic genome. Nature. 2004;431:99-104

50. Lee TI, Rinaldi NJ, Robert F, Odom DT, Bar-Joseph Z, Gerber GK, et al. Transcriptional regulatory networks in Saccharomyces cerevisiae. Science. 2002;298:799-804.

51. Workman CT, Mak HC, McCuine S, Tagne JB, Agarwal M, Ozier O, et al. A systems approach to mapping DNA damage response pathways. Science. 2006:312:1054-9.

52. Zhu C, Byers KJ, McCord RP, Shi Z, Berger MF, Newburger DE, et al. High-resolution DNA-binding specificity analysis of yeast transcription factors. Genome Res. 2009;19:556-66.

53. Vyas VK, Berkey CD, Miyao T, Carlson M. Repressors Nrg1 and Nrg2 regulate a set of stress-responsive genes in Saccharomyces cerevisiae. Eukaryot Cell. 2005:4:1882-91.

54. Kuchin S, Vyas VK, Carlson M. Snf1 protein kinase and the repressors Nrg1 and Nrg2 regulate FLO11, haploid invasive growth, and diploid pseudohyphal differentiation. Mol Cell Biol. 2002;22:3994-4000.

55. Mayordomo I, Estruch F, Sanz P. Convergence of the target of rapamycin and the Snf1 protein kinase pathways in the regulation of the subcellular localization of Msn2, a transcriptional activator of STRE (Stress Response Element)-regulated genes. J Biol Chem. 2002;277:35650-6.

56. Mira NP, Becker JD, Sá-Correia I. Genomic expression program involving the Haa1p-regulon in Saccharomyces cerevisiae response to acetic acid. OMICS. 2010;14:587-601.

57. Fernandes AR, Mira NP, Vargas RC, Canelhas I, Sá-Correia I. Saccharomyces cerevisiae adaptation to weak acids involves the transcription factor Haa1p and Haa1p-regulated genes. Biochem Biophys Res Commun. 2005;337:95-103.

58. Magasanik B. Ammonia assimilation by Saccharomyces cerevisiae. Eukaryot Cell. 2003;2:827-9.

59. Ding MZ, Wang $X$, Liu W, Cheng JS, Yang Y, Yuan YJ. Proteomic research reveals the stress response and detoxification of yeast to combined inhibitors. PLoS One. 2012;7:e43474

60. Mira NP, Palma M, Guerreiro JF, Sá-Correia I. Genome-wide identification of Saccharomyces cerevisiae genes required for tolerance to acetic acid. Microb Cell Fact. 2010;9:79.

61. Hess DC, Lu W, Rabinowitz JD, Botstein D. Ammonium toxicity and potassium limitation in yeast. PLOS Biol. 2006:4:e351.
62. Bayer TS, Hoff KG, Beisel CL, Lee JJ, Smolke CD. Synthetic control of a fitness tradeoff in yeast nitrogen metabolism. J Biol Eng. 2009;3:1.

63. Wegele H, Müller L, Buchner J. Hsp70 and Hsp90-a relay team for protein folding. Rev Physiol Biochem Pharmacol. 2004;151:1-44.

64. Verghese J, Abrams J, Wang Y, Morano KA. Biology of the heat shock response and protein chaperones: budding yeast (Saccharomyces cerevisiae) as a model system. Microbiol Mol Biol Rev. 2012;76:115-58.

65. Jones GW, Masison DC. Saccharomyces cerevisiae Hsp70 mutations affect $[\mathrm{PSI}+]$ prion propagation and cell growth differently and implicate $\mathrm{Hsp} 40$ and tetratricopeptide repeat cochaperones in impairment of [PSI+]. Genetics. 2003;163:495-506.

66. Loovers HM, Guinan E, Jones GW. Importance of the Hsp70 ATPase domain in yeast prion propagation. Genetics. 2007;175:621-30.

67. Duncan K, Edwards RM, Coggins JR. The pentafunctional arom enzyme of Saccharomyces cerevisiae is a mosaic of monofunctional domains. Biochem J. 1987;246:375-86

68. Bauer BE, Rossington D, Mollapour M, Mamnun Y, Kuchler K, Piper PW. Weak organic acid stress inhibits aromatic amino acid uptake by yeast, causing a strong influence of amino acid auxotrophies on the phenotypes of membrane transporter mutants. Eur J Biochem. 2003;270:3189-95.

69. Yoshikawa K, Tanaka T, Furusawa C, Nagahisa K, Hirasawa T, Shimizu H. Comprehensive phenotypic analysis for identification of genes affecting growth under ethanol stress in Saccharomyces cerevisiae. Fems Yeast Research. 2009;9:32-44.

70. Stambuk BU, Panek AD, Crowe JH, Crowe LM, de Araujo PS. Expression of high-affinity trehalose-H+ symport in Saccharomyces cerevisiae. Biochim Biophys Acta. 1998;1379:118-28

71. Jules M, Guillou V, Francois J, Parrou JL. Two distinct pathways for trehalose assimilation in the yeast Saccharomyces cerevisiae. Appl Environ Microbiol. 2004;70:2771-8

72. Babrzadeh F, Jalili R, Wang C, Shokralla S, Pierce S, Robinson-Mosher A, et al. Whole-genome sequencing of the efficient industrial fuel-ethanol fermentative Saccharomyces cerevisiae strain CAT-1. Mol Genet Genomics. 2012;287:485-94.

73. Carreto L, Eiriz MF, Gomes AC, Pereira PM, Schuller D, Santos MA. Comparative genomics of wild type yeast strains unveils important genome diversity. BMC Genomics. 2008;9:524.

74. Dunn B, Levine RP, Sherlock G. Microarray karyotyping of commercial wine yeast strains reveals shared, as well as unique, genomic signatures. BMC Genomics. 2005;6:53.

75. Ask M, Mapelli V, Hock H, Olsson L, Bettiga M. Engineering glutathione biosynthesis of Saccharomyces cerevisiae increases robustness to inhibitors in pretreated lignocellulosic materials. Microb Cell Fact. 2013;12:87.

76. Stephen DW, Jamieson DJ. Amino acid-dependent regulation of the Saccharomyces cerevisiae GSH1 gene by hydrogen peroxide. Mol Microbiol. 1997;23:203-10.

77. Nijkamp JF, van den Broek $M$, Datema $E$, de Kok S, Bosman L, Luttik MA et al. De novo sequencing, assembly and analysis of the genome of the laboratory strain Saccharomyces cerevisiae CEN.PK113-7D, a model for modern industrial biotechnology. Microb Cell Fact. 2012;11:36.

78. Langmead B, Trapnell C, Pop M, Salzberg SL. Ultrafast and memory-efficient alignment of short DNA sequences to the human genome. Genome Biol. 2009;10:R25.

79. Li H, Ruan J, Durbin R. Mapping short DNA sequencing reads and calling variants using mapping quality scores. Genome Res. 2008;18:1851-8.

80. Nakamura K, Oshima T, Morimoto T, Ikeda S, Yoshikawa H, Shiwa Y, et al Sequence-specific error profile of Illumina sequencers. Nucleic Acids Res. 2011;39:e90.

81. Oyola SO, Otto TD, Gu Y, Maslen G, Manske M, Campino S, et al. Optimizing Illumina next-generation sequencing library preparation for extremely ATbiased genomes. BMC Genomics. 2012;13:1.

82. Xie C, Tammi MT. CNV-seq, a new method to detect copy number variation using high-throughput sequencing. Bmc Bioinformatics. 2009;10:80.

83. Artimo $\mathrm{P}$, Jonnalagedda $\mathrm{M}$, Arnold $\mathrm{K}$, Baratin $\mathrm{D}$, Csardi $\mathrm{G}$, de Castro $\mathrm{E}$, et al. ExPASy: SIB bioinformatics resource portal. Nucleic Acids Res. 2012;40:W597-603.

84. Baggerly KA, Deng L, Morris JS, Aldaz CM. Differential expression in SAGE: accounting for normal between-library variation. Bioinformatics. 2003:19:1477-83.

85. da Huang W, Sherman BT, Lempicki RA. Systematic and integrative analysis of large gene lists using DAVID bioinformatics resources. Nat Protoc. 2009:4:44-57. 
86. Merico D, Isserlin R, Bader GD. Visualizing gene-set enrichment results using the Cytoscape plug-in enrichment map. Methods Mol Biol. 2011;781:257-77.

87. Merico D, Isserlin R, Stueker O, Emili A, Bader GD. Enrichment map: a network-based method for gene-set enrichment visualization and interpretation. PLoS One. 2010;5:e13984.

88. Cherry JM, Hong EL, Amundsen C, Balakrishnan R, Binkley G, Chan ET, et al. Saccharomyces Genome Database: the genomics resource of budding yeast. Nucleic Acids Res. 2012;40:D700-5.

89. Abdulrehman D, Monteiro PT, Teixeira MC, Mira NP, Lourenco AB, dos Santos SC, et al. YEASTRACT: providing a programmatic access to curated transcriptional regulatory associations in Saccharomyces cerevisiae through a web services interface. Nucleic Acids Res. 2011;39:D136-40.

90. Monteiro PT, Mendes ND, Teixeira MC, d'Orey S, Tenreiro S, Mira NP, et al. YEASTRACT-DISCOVERER: new tools to improve the analysis of transcriptional regulatory associations in Saccharomyces cerevisiae. Nucleic Acids Res. 2008;36:D132-6.

91. Teixeira MC, Monteiro P, Jain P, Tenreiro S, Fernandes AR, Mira NP, et al. The YEASTRACT database: a tool for the analysis of transcription regulatory associations in Saccharomyces cerevisiae. Nucleic Acids Res. 2006:34:D446-51.

92. Russell DW, Jensen R, Zoller MJ, Burke J, Errede B, Smith M, et al. Structure of the Saccharomyces cerevisiae $\mathrm{HO}$ gene and analysis of its upstream regulatory region. Mol Cell Biol. 1986;6:4281-94.

\section{Submit your next manuscript to BioMed Central and take full advantage of:}

- Convenient online submission

- Thorough peer review

- No space constraints or color figure charges

- Immediate publication on acceptance

- Inclusion in PubMed, CAS, Scopus and Google Scholar

- Research which is freely available for redistribution 